\title{
Detection of $C$. difficile common antigen: comparison of methods
}

\author{
Maria Giuliana Brunelli, Sabrina Tamburelli, Gian Lorenzo Molinari, Stefano Andreoni \\ Laboratorio di Microbiologia e Virologia, Azienda Ospedaliero-Universitaria “Maggiore della Carità" di Novara
}

Key words: $C$. difficile, Common antigen (GDH), Antibiotic-associated pseudomembranous colitis

\section{La ricerca di antigene comune di $C$. difficile: confronto fra metodi}

\section{SUMMARY}

In order to identify and define a diagnostic algorithm for the diagnosis of $C$. difficile infection, a comparative study was carried out at the Microbiology Laboratory of "Maggiore della Carità" Hospital in Novara. We compared the system currently in use in the laboratory "TECHLAB C.difficile Quik Chek Complete (Inverness Medical, USA)", an immunoenzymatic assay for the simultaneous detection of $C$. difficile common antigen (GDH) and Toxins A\&B, with the new ImmunoCard Clostridium difficile GDH (Meridian Bioscience, USA), which identifies the C. difficile Common Antigen GDH. The results proved identical between the two assays: of 100 samples, 82 resulted negative with both tests, 18 were GDH positive with both tests. Out of the $18 \mathrm{GDH}$ positives, 9 resulted positive for the Toxins portion of the TechLab test.

\section{INTRODUZIONE}

Clostridium difficile, bacillo Gram positivo, anaerobio, sporigeno, largamente diffuso nel suolo, presente nel tratto intestinale degli animali e in quello umano, può essere responsabile di patologie di carattere infettivo (ICD), di differente entità, generalmente in seguito a trattamento con antibiotici (2).

Il range di quadri clinici va dalle forme di colonizzazione asintomatica, a forme di diarrea di entità media (AAC: antibiotic-associated diarrhea), a sindromi più consistenti che includono dolore addominale, febbre e leucocitosi (CDAD: Clostridium difficile associated disease), a forme molto gravi (AAPMC: Antibiotic-Associated Pseudomembranous Colitis) (6), fino ad arrivare alle forme fulminanti con megacolon tossico o empiema, perforazione intestinale, peritonite, sepsi, shock che possono costituire causa diretta o indiretta di morte.

Le ICD sono ormai riconosciute da più parti come la principale causa di diarrea infettiva in ambito ospedaliero e in quelle strutture in cui si pratica assistenza sanitaria (strutture riabilitative, per anziani).

Sebbene i pazienti anziani ospedalizzati siano il principale gruppo a rischio d'infezione, sono in aumento segnalazioni di coinvolgimento di popolazioni più giovani senza contatti recenti con strutture ospedaliere o in assenza di trattamenti antibiotici, così come bambini e donne in gravidanza.
In seguito all'incremento d'incidenza o comunque di severità, in relazione anche alla comparsa di ceppi ipervirulenti, le ICD hanno conquistato un posto sempre più rilevante nel dibattito medico e scientifico, risultando un importante problema di sanità pubblica, anche in termini di costi economici (3).

L'IDSA (Infectious Diseases Socety of America) e altre società scientifiche hanno definito linee guida per la diagnosi di ICD. Questa si basa sulla ricerca di $C$. difficile e/o di suoi antigeni, tossine o acidi nucleici su filtrato fecale.

A causa delle limitazioni inerenti a ciascun metodo e per cercare di combinare la rapidità di risposta con una buona sensibilità, la Letteratura Internazionale e quella Nazionale suggeriscono algoritmi diagnostici (Schema 1-2) che prevedano step successivi.

Tra questi algoritmi vanno sicuramente considerati quelli che prevedono un primo screening volto alla ricerca di glutammato deidrogenasi (GDH) (il cosiddetto antigene comune) e la successiva verifica, a fronte di un riscontro positivo, della produzione di tossina $\mathrm{A} / \mathrm{B}$, mediante sistemi immunologici e/o molecolari $(1,4,5)$.

Una recente revisione dei principali sistemi presenti in commercio per la rilevazione di antigeni e tossine di $C$. difficile con metodi immunometrici (EIA/IC) ha portato alla conclusione che, considerando la mancanza di differenze sostanziali nelle performances complessive dei differenti kit e l'elevato valore predittivo negativo (VPN) $(>98 \%)$

\section{Corresponding author: Stefano Andreoni}

Lab. Microbiologia e Virologia

Azienda Ospedaliero Universitaria "Maggiore della Carità" di Novara

E-mail: stefano.andreoni@maggioreosp.novara.it 
dei test del commercio, data una sensibilità rilevata non inferiore al $75 \%$, un risultato negativo permette, in un contesto di prevalenza dell'infezione relativamente bassa, con elevata probabilità di escludere l'infezione.

\section{MATERIALI E METODI}

Nel corso del 2011, è stato condotto, presso il Laboratorio di Microbiologia dell'Azienda Ospedaliera "Maggiore della Carità" di Novara, un confronto tra il sistema in uso "TECHLAB $C$. difficile Quik Chek Complete - Inverness Medical (Princeton-USA)", sistema immunocromatografico in grado di evidenziare l'antigene comune $(\mathrm{GDH})$ di $C$. difficile e contemporaneamente la presenza di tossina $\mathrm{A} / \mathrm{B}$, sistema già oggetto di verifiche e confronti in termini di sensibilità e specificità (7) e un nuovo sistema "ImmunoCard Clostridium difficile GDH"Meridian Bioscence, in grado di evidenziare la presenza del solo antigene comune (GDH) di $C$. difficile (8).

Campioni di feci fresche di pazienti ospedalizzati e ambulatoriali, sono stati quindi sottoposti ad indagine per la ricerca di antigene comune di $C$. difficile utilizzando contemporaneamente entrambi i metodi e secondo le indicazioni dei kit commerciali.

Il protocollo operativo prevedeva, la registrazione dei risultati concordanti (ricerca dell'antigene comune positiva e negativa per entrambi i sistemi) e, nel caso di risultati discordanti (positivo/negativo o negativo/positivo), un'ulteriore verifica mediante coltura: partendo sempre da feci fresche, si sarebbe proceduto, dopo shock etanolico di una sospensione fecale e la successiva germinazione delle spore a bagnomaria a $56^{\circ} \mathrm{C}$, alla semina su Schaedler agar in condizioni di anaerobiosi.

Le colonie eventualmente sviluppatesi, con aspetto compatibile con Clostridium (osservazione microscopica dopo colorazione di Gram), sarebbero state sottoposte a saggi d'identificazione biochimico-assimilativa e spettrofotometrica (Rapid ID32ANA - bioMérieux; MALDI TOF - Bruker Daltonik). Confermata la presenza di $C$. difficile si sarebbe proceduto, attraverso una sospensione in substrato liquido, ad un ulteriore saggio con i due sistemi.

\section{RISULTATI}

Nel periodo Maggio 2011 - Settembre 2011, 100 campioni fecali d'isolamento clinico, sono stati sottoposti alla ricerca di antigene comune di $C$. difficile utilizzando in contemporanea i due metodi in oggetto. Come riportato nella Tabella 1, i risultati hanno evidenziato una completa concordanza tra i due sistemi: 82 campioni sono risulta- ti negativi e 18 sono risultati positivi per antigene comune di $C$. difficile utilizzando tanto il sistema TECHLAB $C$. difficile Quik Chek Complete quanto il sistema ImmunoCard Clostridium difficile GDH. Dei 18 campioni positivi, 9 sono risultati anche produttori di tossina A/B come evidenziato dal sistema Inverness.

TABELLA I. Rilevazione di antigene comune di C. difficile.

\begin{tabular}{lcc}
\hline & CD Chek Complete & CD GDH \\
\hline POSITIVI & 18 & 18 \\
\hline NEGATIVI & 82 & 82 \\
\hline TOXIN A/B + & 9 & $*$ \\
\hline TOXIN A/B - & 9 & $*$ \\
\hline
\end{tabular}

* non determinabile

\section{DISCUSSIONE}

Dalle nostre osservazioni si conferma la validità del sistema ImmunoCard Clostridium difficile GDH come test di screening per la ricerca di GDH su campioni fecali per la diagnosi di infezione da C. difficile. Come suggerito da numerosi algoritmi diagnostici proposti da Società scientifiche (AMCLI, SIMPIOS, IDSA, HPA, ASM), riferibili alle ICD, l'utilizzo di sistemi volti alla ricerca dell'antigene comune (GDH) come test di screening iniziale, risulta essere il più indicato in relazione alla buona sensibilità del test stesso, all'elevato VPN, alla considerazione, non trascurabile, che l'antigene risente meno rispetto alle tossine della conservazione del campione e, non ultimo, dal contenimento dei costi.

In base a queste considerazioni e in seguito alle nostre osservazioni, il sistema ImmunoCard Clostridium difficile GDH può rappresentare una valida opportunità ai fini diagnostici. Naturalmente l'utilizzo di un test di screening deve prevedere, in caso di positività, la conferma della presenza o meno di uno stipite tossinogenico, mediante test immunoenzimatici, mediante test di citotossicità $\mathrm{o}$ direttamente mediante metodi molecolari, sistemi che garantiscano VPP superiori.

\section{BIBLIOGRAFIA}

1. A Practical Guidance Document for the laboratory Detection of Toxigenic Clostridium difficile. American Society for Microbiology - September 21, 2010

2. Bartlett JG, Chang TW, Gurwith M, et al. Antibioticassociated pseudomembranous colitis due to toxinproducing clostridia. N Engl J Med 1978; 298: 531-4.

3. Bartlett JG, Gerding DN. Clinical recognition and diagnosis of Clostridium difficile infection. Clin Infect Dis 2008; 46 (Suppl.1): S12-18.

4. Cohen SH, Gerding DN, Johnson S, et al. Clinical practice guidelines for Clostridium difficile infection in adults: 2010 update by the society for healthcare epidemiology of America (SHEA) and the infectious 
diseases society of America (IDSA). Infect Control Hosp Epidemiol. 2010; 31(5): 431-55.

5. Eastwood K, Else P, Charlett A, Wilcox M. Comparison of Nine Commercially Available Clostridium difficile Toxin Detection Assays, a RealTime PCR Assay for C. difficile $t c d B$, and a Glutamate Dehydrogenase Detection Assay to Cytotoxin Testing and Cytotoxigenic Culture Methods. Journal of Clinical Microbiology, October 2009; 47: 3211-7.

6. Larson HE, Price AB, Honour P, Borriello SP. Clostridium difficile and the aetyology of pseudomembranous colitis. Lancet 1978; 1: 1063-6.

7. Sharp SE, Ruden LO, Pohl JC, Hatcher PA, Jayne LM, Ivie WM. Evaluation of the $C$. difficile. Quik Chek Complete Assay, a new glutamate dehydrogenase and $\mathrm{A} / \mathrm{B}$ toxin cambination lateral flow assay for use in rapid, simple diagnosis of Clostridium difficile disease. J Clin Microbiol. 2010; 48(6): 2082-6.

8. Shetty N, Wren MWD, Coen PG. The role of glutamate dehydrogenase for the detection of Clostridium difficile in faecal samples: a meta-analysis. Journal of Hospital Infection. 2011; 77: 1-6. 